\title{
Modifications of Ti-6Al-4V Surfaces by Direct-Write Laser Machining of Linear Grooves
}

\author{
Joseph P. Ulerich, Lara C. Ionescu, Jianbo Chen, Winston O. Soboyejo, Craig B. Arnold* \\ Department of Mechanical and Aerospace Engineering, Princeton University, Princeton NJ 08540, \\ USA
}

\begin{abstract}
As patients who receive orthopedic implants live longer and opt for surgery at a younger age, the need to extend the in vivo lifetimes of these implants has grown. One approach is to pattern implant surfaces with linear grooves, which elicit a cellular response known as contact guidance. Lasers provide a unique method of generating these surface patterns because they are capable of modifying physical and chemical properties over multiple length scales. In this paper we explore the relationship between surface morphology and laser parameters such as fluence, pulse overlap (translation distance), number of passes, and machining environment. We find that using simple procedures involving multiple passes it is possible to manipulate groove properties such as depth, shape, sub-micron roughness, and chemical composition of the Ti-6Al-4V oxide layer. Finally, we demonstrate this procedure by machining several sets of grooves with the same primary groove parameters but varied secondary characteristics. The significance of the secondary groove characteristics is demonstrated by preliminary cell studies indicating that the grooves exhibit basic features of contact guidance and that the cell proliferation in these grooves are significantly altered despite their similar primary characteristics. With further study it will be possible to use specific laser parameters during groove formation to create optimal physical and chemical properties for improved osseointegration.
\end{abstract}

Keywords: laser surface texturing, laser micromachining, cell growth, titanium

\section{INTRODUCTION}

Modern surgical techniques enable the replacement or supplement of worn bones and joints with biomedical implants in order to avoid the lifestyle limitations of bone degradation. Unfortunately, like human bone itself, the interface between these implants and the human skeletal structure degrades over time. It is not uncommon for implant loosening to cause significant pain and malfunction after 10-15 years requiring dangerous implant retrievals and difficult revision surgeries to remove or reattach surgical implants. Problems are so common that younger, more active, or heavier patients are often advised against surgeries and forced to live with chronic pain for years because doctors fear the patients will outlive their implants. Because of these failures, efforts to increase cell adhesion at this biomaterial interface have spread throughout the field of bioengineering.

Current research focuses on the initial stages of cell adhesion when young osteoblast cells lay the groundwork for cell adhesion. During healing, new osteoblast cells must gather, adhere, and proliferate in the empty regions between the implant and original bone to 'seal' the implant into place. The early activities of new osteoblast cells that migrate to the surgery site lay the groundwork for mature bone cells that will exist in that region in the future [1]. Thus, coaxing or controlling young osteoblast cells to both attach properly and grow in 'natural' ways can increase the chances of successful osseointegration.

There are several techniques used to alter the attachment scheme of young osteoblast cells and aid in successful osseointegration. First, material selection plays a large role in implant success since non-biocompatible materials will cause macrophage proliferation at the bioimplant surface, resulting in swelling, irritation, and bone loss. Titanium is one of few completely biocompatible metals and for this reason, coupled with its excellent fracture toughness, fatigue resistance, and Young's Modulus near that of bone, Ti-6Al-4V (Ti64) is the most common metal biomaterial used in dental and hip implants.

* Corresponding Author: E-mail: cbarnold@princeton.edu, phone: 1-609-258-0250

Photon Processing in Microelectronics and Photonics VI, edited by Craig B. Arnold, Tatsuo Okada, Michel Meunier, Andrew S. Holmes, David B. Geohegan, Frank Träger, Jan J. Dubowski, Proc. of SPIE Vol. 6458, 645819, (2007) 0277-786X/07/\$18 - doi: 10.1117/12.713964 
Once an appropriate material is selected for a bioimplant, surface engineering is used to increase cell adhesion specifically at the cell-surface interface. One common strategy is to introduce chemical stimulants at the surface to increase surface wetability or the cells affinity to the surface. A second strategy involves modifying the surface topography of the interface to increase adhesion strength.

The topography of a biomaterial interface is important at multiple length scales. Individual cell microfilaments and microtubules interact to form focal adhesion complexes at the nanoscale, cells and individual cell features align at the micron and submicron scales, and full organs and tissues interact with larger surface contours at the macroscale. Currently, implant surfaces are roughened through randomized processes such as sand blasting to encourage cell growth and improve osseointegration. Such techniques are relatively inexpensive and easy to perform on complex surfaces [2, 3], but cells that grow on these surface typically do so equiaxially and tend to be randomly oriented

In contrast, contact guidance, where cells align with micron-sized linear grooves patterned onto a surface has the potential to improve osseointegration and increase longevity in implants for a number of reasons. From a mechanical perspective, the grooves can physically interlock the implant with new bone, improving stability and increasing longevity [4-8]. Also, the increase in surface area provides more opportunities for focal attachment. From a biological perspective, contact guidance may elicit a number of cell responses that promote healthy regeneration of bone and tissue due to the cells elongating within the grooves. Since bone consists of sheets of parallel cells, initiating bone healing with parallel cells may improve the healing process [9]. Also, cells grown on substrates with linear grooves exhibit organized regrowth, possibly decreasing scar tissue formation during healing [10].

Generating micron-sized grooves rapidly and with precision on non-planar surfaces is not easily done using traditional lithographic patterning. Recent developments in photolithography for applications in microelectronics processing allow for the creation of grooves on many polymeric substrates [11]. These surfaces can then be coated with various materials for in vitro cell testing. While photolithography allows for the precise study of topology on a single size scale, other techniques, such as lasers direct writing, can more easily be applied to implant surfaces and can generate features on multiple length scales [12]. Lasers provide excellent controllability, agility, and efficiency at removing small amounts of metal from a surface. However, local heating during laser machining results in more subtle physical and chemical changes that may influence cell behavior.

To begin to understand the effects of laser irradiation on biological surfaces, large-area masking techniques and excimer laser generated groove geometries have been examined [10]. They found features such as molten packets, bubbles, and cracks that had formed in the grooves. While this type of work provides evidence of the surface changes from laser machining, a large-area masking technique cannot be applied to the contoured surfaces of an implant. Instead, a direct-write processing technique can be used where small diameter pulses $(\sim 10 \mu \mathrm{m})$ sequentially administered remove material to generate grooves.

In this paper, we present results using laser direct write patterning to create multiscale features on Ti64. Primary and secondary groove structures are controlled through the incident laser parameters and characterized for their morphological properties. Preliminary results for osteoblast growth on such surfaces are presented demonstrating the importance of multiscale texturing.

\section{PROCEDURE}

A laser direct-write system composed of a pulsed frequency tripled $\mathrm{Nd}: \mathrm{YVO}_{4}$ laser $(355 \mathrm{~nm}), \mathrm{UV}$ corrected focusing objective, and a three axis computer controlled translation system were used for this study. Ti64 discs were polished using a series of polishing clothes (400 grit, 600 grit, 800 grit, 1200 grit) culminating with 0.05 micron colloidal silica on a synthetic velvet pad. The purpose of polishing the samples prior to laser micromachining was to create a uniform surface such that the morphological changes induced by the laser could be measured. Laser machining parameters that were varied in this study included laser energy (between 0 and $300 \mu \mathrm{J})$, translation distance $(\Delta$, the distance moved between each laser pulse, 2-10 $\mu \mathrm{m}$ ), number of passes over the same groove (1-2 passes), and machining environment (air, water, and silicon oil). The laser focusing parameters remained fixed in this study. For the 
purposes of calculating fluence, we assumed a beam diameter of $10 \mu \mathrm{m}$, which was measured at a pulse energy of 50 $\mu \mathrm{J}$.

Sample morphology was characterized using optical microscopy, stylus profilometry, and scanning electron microscopy. A stylus tip radius of $2 \mu \mathrm{m}$ was used, giving us the ability to profile the bottom of the micromachined grooves obtaining depth and roughness parameters. SEM imaging using secondary electrons provided confirmation of the groove parameters and classification of the secondary features formed in the grooves. Cross sectional information was obtained by dicing the Ti64 disks with a diamond saw and repolishing along the fresh cut. Additionally EDS was used to give compositional information about the surface regions in and around the groves.

For cell growth studies, samples were cleaned to remove any loose debris and or biocontaminants generated during the micromachining process. The discs were sanitized and passivated in a series of rinses of nitric acid, ethanol, and distilled water. Human osteosarcoma cells were cultured and allowed to grow for various time periods before being partially dehydrated and observed. Cells without staining were grown for 0.5 or 2 hours and observed by the SEM, while cells stained with rhodamine phalloidin for actin, and primary and secondary vinculin antibodies were grown for 24 hours and observed with a fluorescence imaging microscope.

\subsection{Primary Microscale Groove Parameters}

\section{RESULTS}

The two fundamental primary properties of grooves are the groove depth and groove width, and to a lesser extent the slope of the groove wall. As shown in Fig 1b, the width of grooves as measured between the peaks in the surface structure is not affected significantly by either the number of passes or the distance between pulses. Rather, for fixed focus location, it is a function of the pulse energy. As the peak energy increases, the volume of material above the ablation threshold increases and therefore the width of the groove increases. Simple attempts to model width as a function of laser energy are difficult since the width between ridges includes effects of recast and redeposited species. For the purposes of this study, the width, including the ridges is more appropriate for the cell attachment studies and is therefore included. However, it suffices to say that we can accurately manipulate the width of a groove by modifying only laser energy.

Groove depth is affected by translation distance, laser energy per pulse, and number of passes. We can accurately predict depth of a groove if we assume a simple model for the ablation process that requires the material removed on each pulse is independent of previous pulses. The amount of material removed on each pulse can be
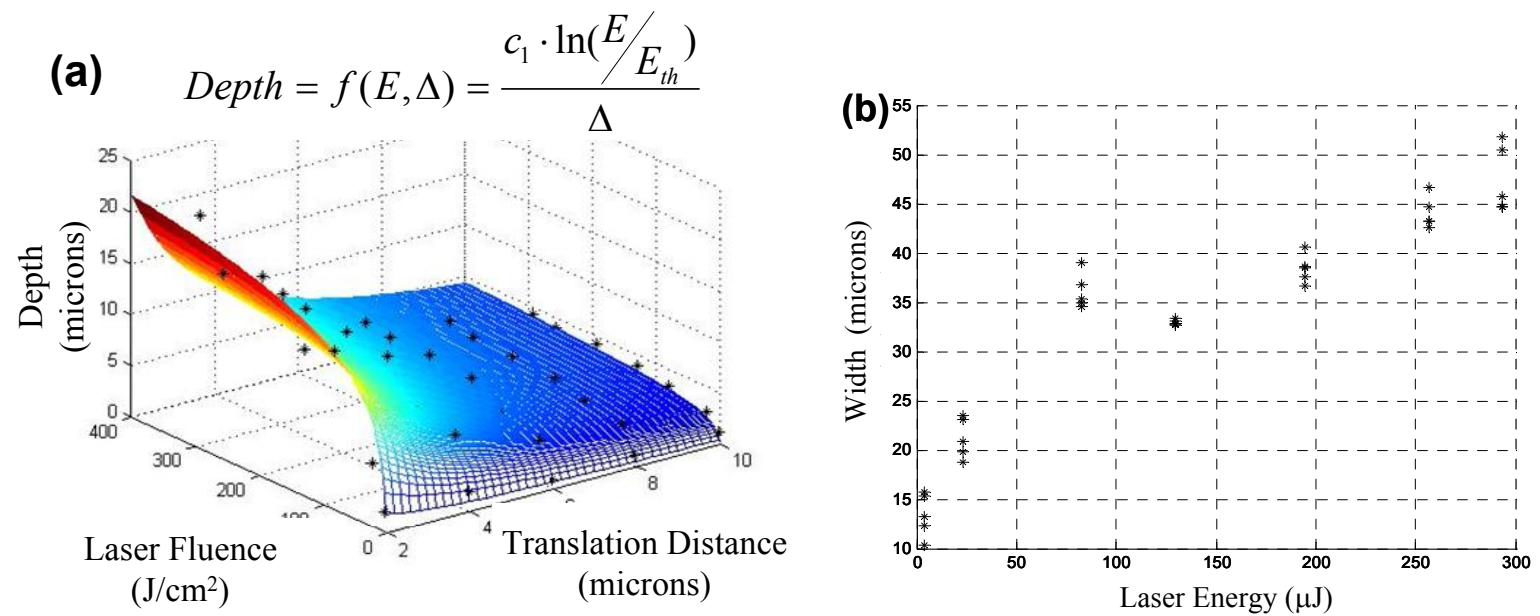

Figure 1: (a) Groove depth measurement as a function of translation distance and laser fluence. 2-d surface shows fit of data points to given equation. (b) Groove width as measured from ridge maximum to ridge maximum as a function of laser energy. 
represented by a standard Beer's law relation. The resulting equation gives the groove depth proportional to the inverse the translation distance $(1 / \Delta)$ and the natural log of pulse energy over some threshold energy needed for ablation $\left(\ln \left(\mathrm{E} / \mathrm{E}_{\mathrm{th}}\right)\right)($ Fig. 1a). Adding a second pass without changing the focal plane tends to increase depth, but it does not double the depth of the groove as would be expected by this simple model. Rather, the effects of pre-existing surface structure and changes in position of focus relative to surface cause a reduction in observed depth increases the depth by only $10-15 \%$.

The slope on edge of the grooves can be observed in cross-sectional microscopy as shown in figure 2 . As the translation distance decreases, the groove depth increases more than the width increase. The result is a groove with steep walls. Conversely, at large translation distance, the groove is shallower and the resulting slopes are lower. Additionally, the laser energy affects both the width and depth of the grooves, but it does not change both with the same functional dependence. Therefore it is possible to gain additional control over the groove wall slope through independent control of laser energy and translation distance.
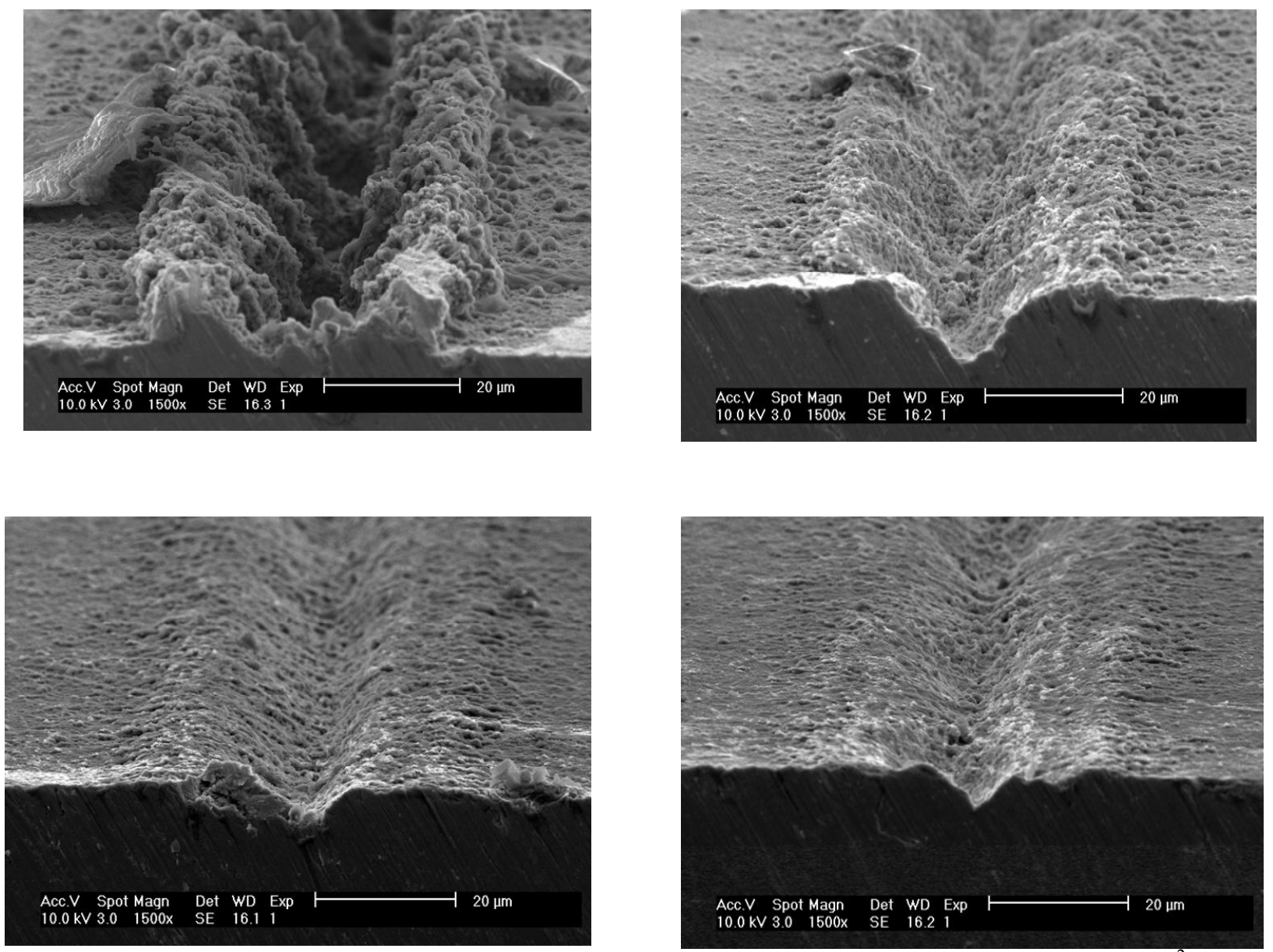

Figure 2: Size and slope of groove walls decreases with increasing translation distance generated at $56 \mathrm{~J} / \mathrm{cm}^{2}$. Upper left shows translation distance of $2 \mu \mathrm{m}$, upper right, $4 \mu \mathrm{m}$, lower left, $6 \mu \mathrm{m}$, and lower right $8 \mu \mathrm{m}$. Scale bar in all images is $20 \mu \mathrm{m}$.

\subsection{Secondary Micro and Submicroscale Features}


Although groove shape and depth are the primary considerations when generating grooves using photolithography, laser processing techniques also create roughness and sub-micron features that can be beneficial for cell proliferation and adhesion. Small-scale features form on the surface of the grooves due to a variety of factors including heating, resolidification, and removal of metal from recoil pressures. The sub-micron roughness seen in Figure 3 is the result of thermal gradients, heat effects, and ejected material leaving the surface. This figure shows that by increasing laser energy we are able to create significantly different submicron surface topographies. Lower energies have a tendency to merely melt the surface with thermocapillarity causing a net change in the surface features. We denote such features as perpendicular ridges and can be seen in Figure 3a. As energy increases, the material begins to experience larger recoil pressures and material is now ablated and redeposited to the surrounding areas with each pulse. This creates features of a significantly smaller length scale and increases surface roughness substantially. These features are denoted parallel microfeatures as they form parallel to the direction of the laser translation and can be seen most clearly in Figure 3d. Decreasing translation distance also has a tendency to increase surface roughness. Closer pulses tend to heat the material more, increasing redeposited material and decreasing the distance between such regions. At very low translation distances, the material retains a significant amount of heat and remains molten for some time after the laser has passed resulting in a molten recast structure (Figure $3 \mathrm{~h}$ ).

A map of the basic secondary features formed at varying machining parameters is shown in figure 4 . In typical commercial implants, surfaces are treated by grit-blasting or plasma spraying to have $\mathrm{R}(\mathrm{a})$ along the groove of order 1-3 $\mu \mathrm{m}$, very similar to the quantities resulting from laser machining. Thus, using direct-write lasers not only generates grooves that elicit contact guidance, but also creates a roughness in the grooves that has been empirically determined by industry.

This dynamic set of machining parameters is capable of creating many sub-micron features including nodules, ripples, ledges and nano-textures. Machining at small translation distances and high energies in some cases can even produce nano-sized pillars, a surprising event considering the beam is at the micro-scale (Figure 5). These pillars sometimes intertwine but generally reach to the air interface at a slight angle, probably related to the direction of the stage motion as flying liquid is left behind. Such pillars resemble self forming nano-columns generated with colloidal lithography by Dalby et al. Human fibroblast cells responded to the topology by forming rounder shapes as compared to a smooth surface, suggesting fewer focal adhesion points. Electron microscopy revealed that the 50-70 nm filopodia

\section{Roughness Increases}
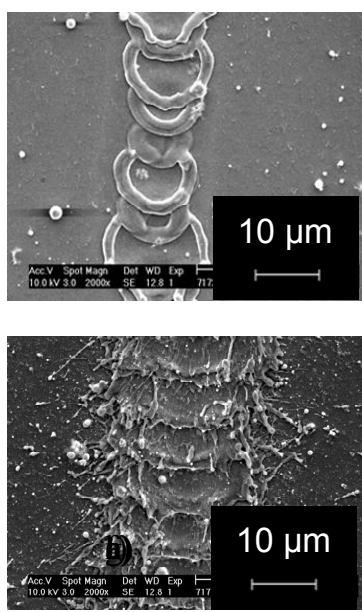

b)

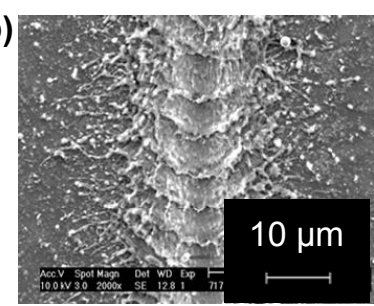

f)

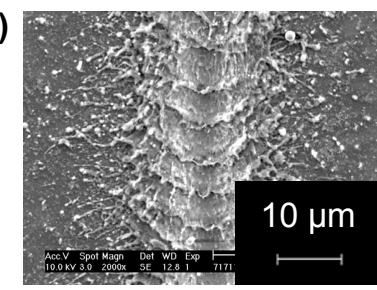

c)

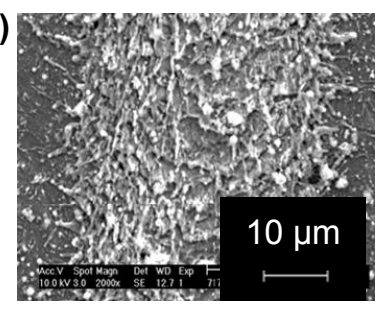

g)

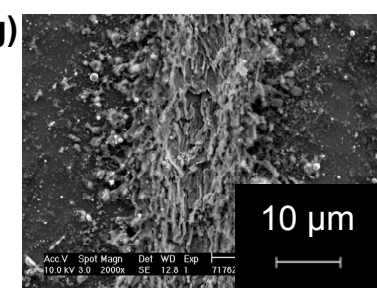

d)

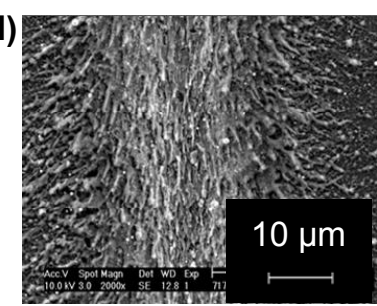

h)

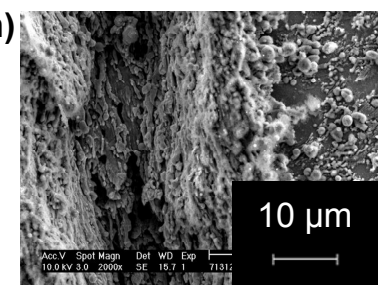

Figure 3: On top, the effect of pulse energy demonstrated by grooves cut with translation distance of $6 \mu \mathrm{m}$ and Pulse energies of a) $3.6 \mu \mathrm{J}$, b) $23 \mu \mathrm{J}$, c) $82 \mu \mathrm{J}$, and d) $130 \mu \mathrm{J}$. On the bottom, the effect of translation distance demonstrated by grooves cut with pulse energy of $23 \mu \mathrm{J}$ and translation distances of e) $10 \mu \mathrm{m}$, f) $8 \mu \mathrm{m}, \mathrm{g}) 6 \mu \mathrm{m}$, and h) $4 \mu \mathrm{m}$. 


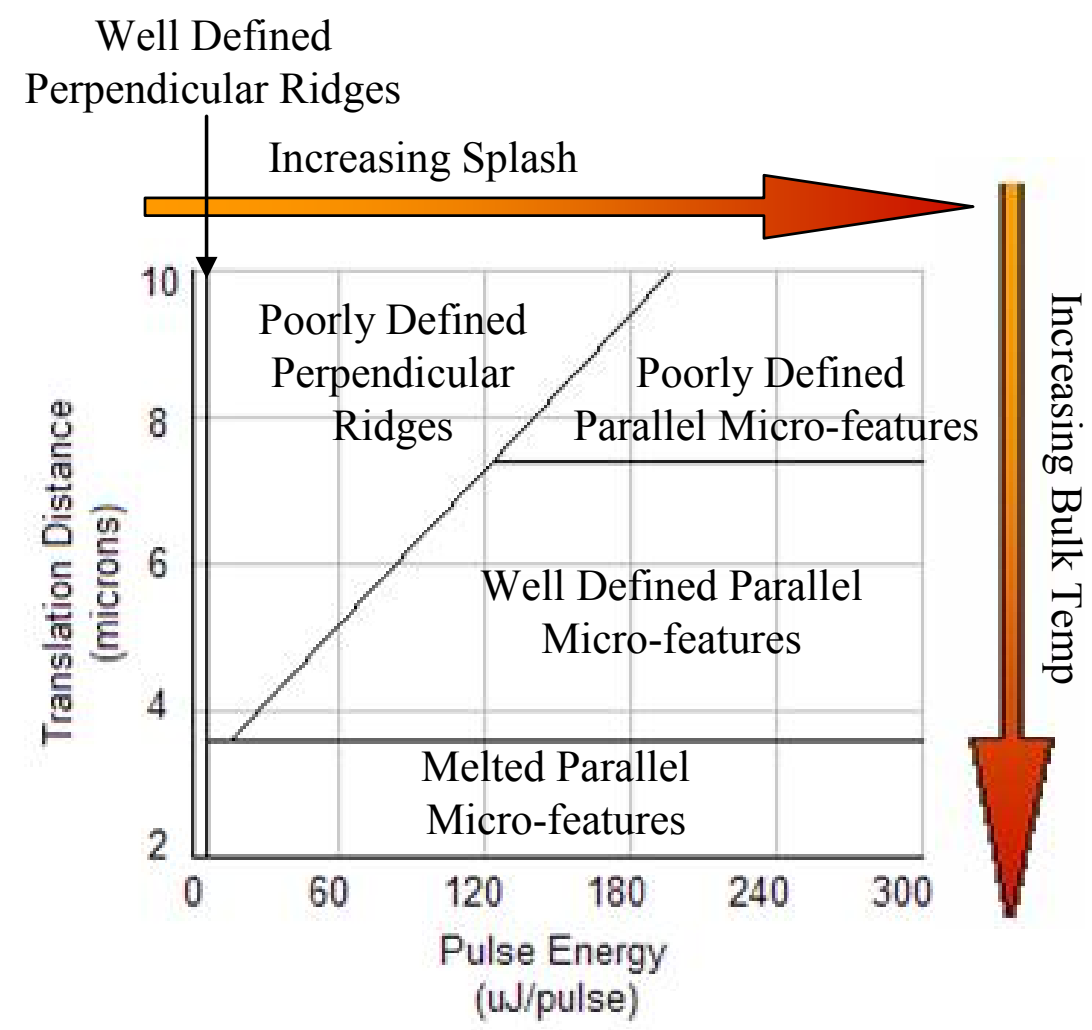

Figure 4: Map of basic features and under the machining parameters under which they are created

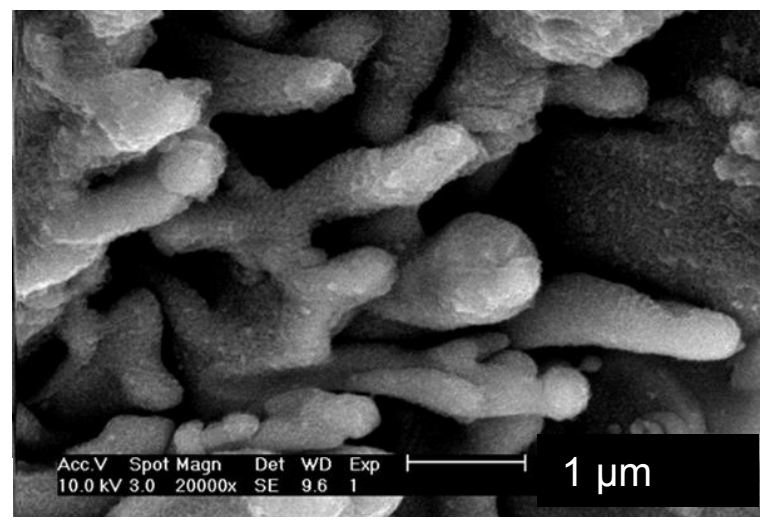

Figure 5: Nanoscale pillars formed pulses with energy of $56 \mathrm{~J} / \mathrm{cm}^{2}$ and a translation distance of $2 \mu \mathrm{m}$

interacted frequently with the nano-columns [13]. Similar features were formed in silicon by Padraza with a nanosecond laser [14] and by Mazur with a femtosecond laser [15].

Since cooling rates influence the development of morphology, machining under a liquid environment significantly influences secondary groove topography. Our results are not yet capable of fully characterizing liquid environments, but several preliminary studies indicate significant trends. Grooves machined in liquid environments are much smoother and contain bubbles ranging from 10 to $50 \mathrm{~nm}$, formed by vapor evolved near the surface (Figure 6). The ability to create bubbles on the bulk surface provides added opportunity for mechanical interlocking of implant with bone, and creates alternative structures for cell studies. Li et al generated similar bubbles but at higher concentrations using a micro-arc oxidation technique, but these were formed in pure titanium, not Ti-6Al-4V [16]. Larger pores are 
also shown effective in improving osseointegration [17]. However, it is unclear whether the small features created through liquid machining, such as the tops of the bubbles, could withstand the implantation procedure or extended periods of time in vivo. Further studies are necessary to better quantify the robustness of these surfaces and more carefully understand the formations of features such as pores and cracks.

Cracks also formed on the surface of the liquid-machined grooves due to the greater quench rate and development of thermal stress. Cracks have the potential to grow over time from cyclic pressures in the body, possibly leading to implant failure. In order to minimize these detrimental features, machining is also performed in silicon oil, which provides a less severe quench. As seen in Figure 6, machining under silicon oil resulted in a decrease in crack formation while maintaining bubble formation.
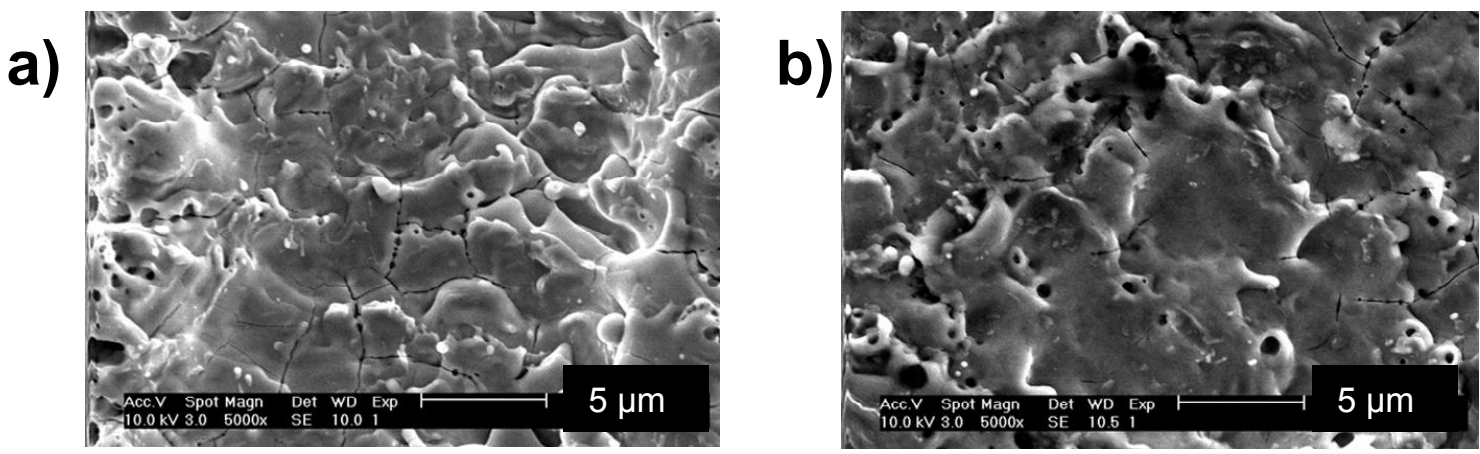

Figure 6: Groove floor after machining under a) water and b) silicon oil. Note the decreased crack formation in silicon oil.

\subsubsection{Chemical Composition}

While physical changes are the primary result of laser texturing, chemical changes also occur and are measurable by EDS. Changes in the chemical composition of the surface may influence how cells attach and react to the metal by changing the way that proteins adsorb or by activating different cellular pathways in the nearby cells. Also, while some work has shown that depleting aluminum on the surface results in better biocompatibility [18], but maintaining high levels of aluminum provides opportunities for bioactivation through chemistry. It was found that a range of chemical compositions could be generated in the surface layer by different machining parameters that could then be tested with cell studies.

One of the most significant results was the loss of aluminum from the surfaces machined in air. The change in aluminum content happened at small translation distances of $2 \mu \mathrm{m}$. Larger translation distances did not lead to appreciable changes in surface chemistry, irrespective of laser fluence. Also, multiple passes did not lead to appreciable changes in chemical content. This is consistent with a transient molten region at the surface that preferentially evaporates aluminum due to its high vapor pressure. In contrast, under liquid environments, the depletion of aluminum did not occur, providing an opportunity for combining laser-created grooves with bioactivation of the surface.

When the grooves were spaced close enough that a sinusoidal morphology is observed, EDS revealed that the ridges actually increased in aluminum concentration compared to the grooves or the original surface. Once again, this is consistent with the formation of a molten pool in the bottom of the groove from which aluminum evaporates and is redeposited on the cooler ridge regions. 

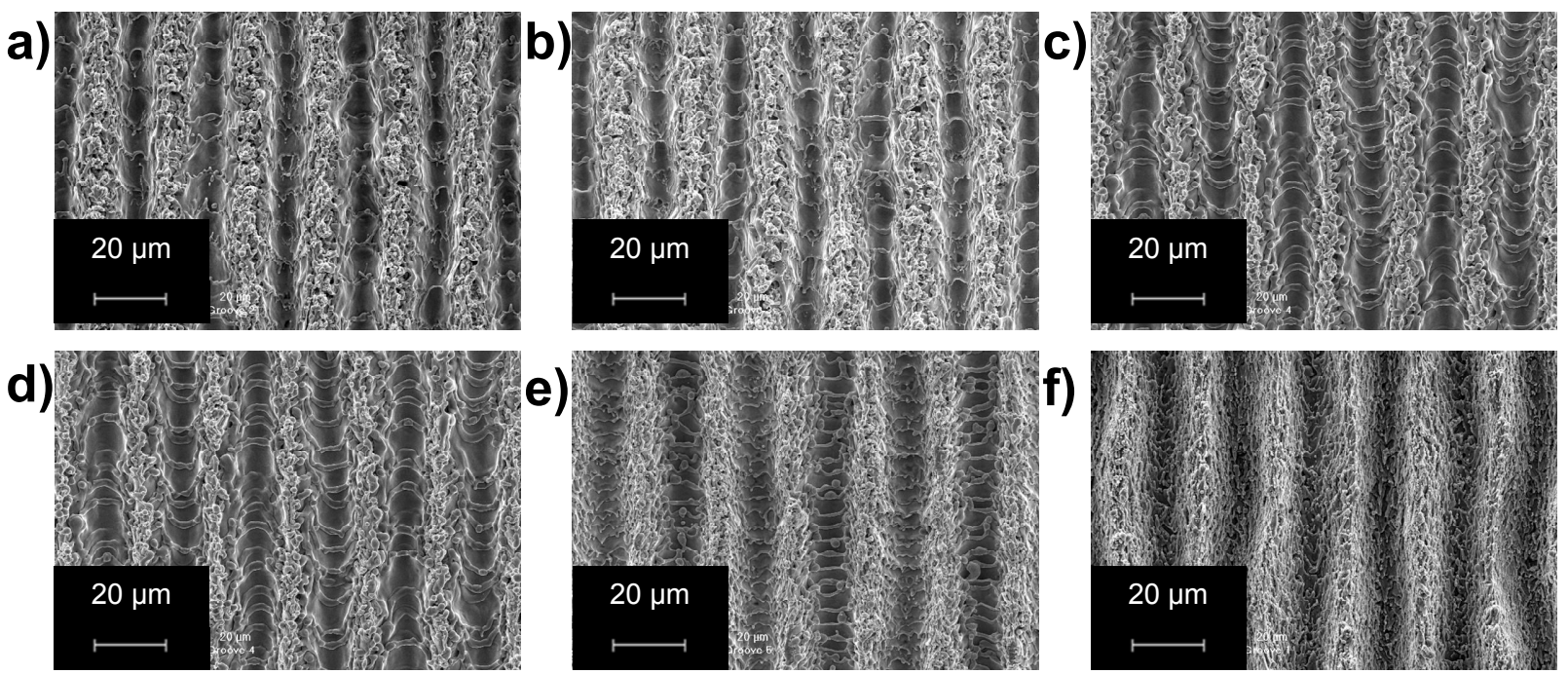

Figure 7: Demonstrating the effect of translation distance, the top grooves have final pass energy of $5 \mu \mathrm{J} / \mathrm{pulse}$ and translation distances of a) $10 \mu \mathrm{m}$, b) $8 \mu \mathrm{m}$, and c) $6 \mu \mathrm{m}$. Demonstrating the effect of final pass pulse energy, the lower grooves have final pass translation distances of 4 or $6 \mu \mathrm{m}$ and energy of d) $5 \mu \mathrm{J} / \mathrm{pulse}$, e) $14 \mu \mathrm{J} / \mathrm{pulse}$, and f) $39 \mu \mathrm{J} / \mathrm{pulse}$.

\section{Groove Engineering}

By combining the results of primary groove patterns and secondary surface texturing, it is possible to design grooves with specific properties. Initial high energy passes create approximate groove dimensions. The laser energy sets approximate groove width, while translation distance and number of passes create a specific depth. Next, lower energy pulses manipulate the secondary submicron features of the grooves without significantly altering their primary characteristics. Secondary features perpendicular to the groove are created by very low energy pulses and their spacing can be manipulated with translation distance, while the frequency and feature sizes parallel to the groove can be manipulated using final pass laser pulse energy. Variable ridge spacing and textures are demonstrated in Figure 7 for six grooves of comparable primary groove characteristics to demonstrate the effect of both translation distance and pulse energy.

\subsection{Cell Alignment}

Initial studies using the laser generated grooves demonstrate that these direct write laser machined grooves do in fact lead to contact guidance for osteoblast cells (Figure 8a). Cells placed over grooves surfaces exhibited both a greater cell density and a greater degree of cell alignment than those on the original surface. However, the primary groove structure alone is not sufficient to determine the degree two which the cells proliferate on the surface. In figure $8 \mathrm{~b}, \mathrm{c}$, we see cases where the basic primary parameters are similar but the submicron roughness is significantly different. Based on this, we may conclude that the secondary groove textures play an important role in determining the growth.

Secondary studies with the more refined grooves shown in Figure 9 demonstrate more succinctly some important trends in submicroscale roughness characteristics. Grooves with final passes of higher energy and larger roughness tend to have a much larger number of cells spanning multiple grooves. This can increase the transmission of signals from one groove to the next and may be a very beneficial. To maximize the amount of contact guidance as measured by cell alignment and cell stretching along grooves, however, it appears that grooves with features perpendicular to the primary grooves with spacings of 6-8 microns are most beneficial. 

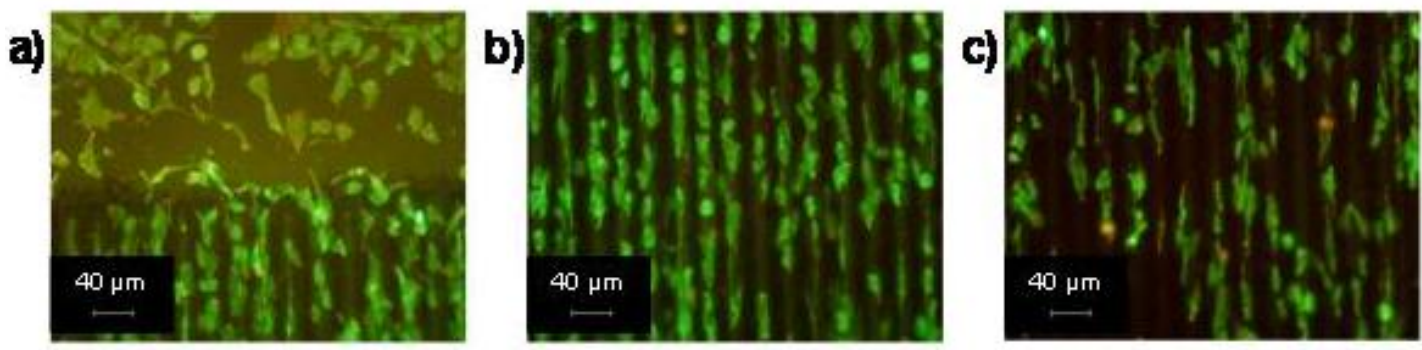

Figure 8: Initial Groove studies demonstrating increased density due to contact guidance a) and variations in cell density based upon submicron roughness changes demonstrating the importance of submicron texturing: b) laser energy $48 \mathrm{~J} / \mathrm{cm}^{2}, 4$ micron, double pass and c) laser energy $28 \mathrm{~J} / \mathrm{cm}^{2}, 2$ micron, single pass
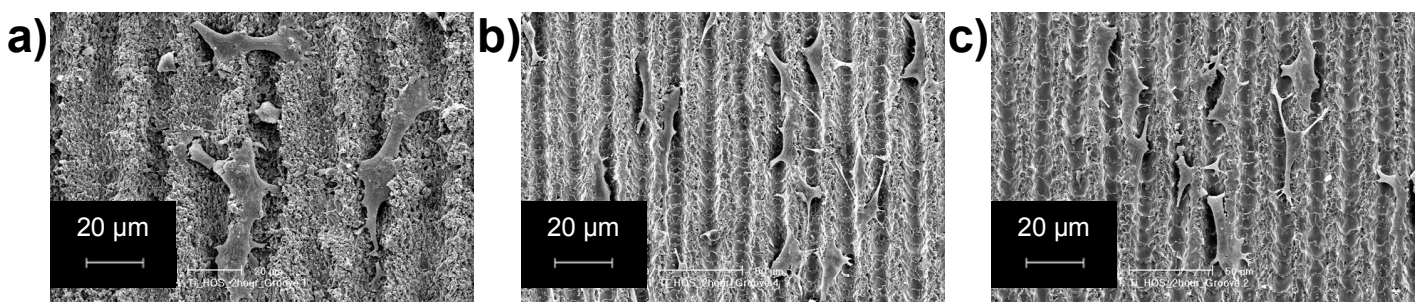

Figure 9: Cell growth after 2 hours on groves sets 10 microns deep and 20 microns wide. Final passes have a) translation distance $4 \mu \mathrm{m}$, pulse energy $39 \mu \mathrm{J} /$ pulse, b) translation distance $6 \mu \mathrm{m}$, pulse energy $5 \mu \mathrm{J} /$ pulse, and c) translation distance $10 \mu \mathrm{m}$, pulse energy $5 \mu \mathrm{J} /$ pulse.

\section{CONCLUSIONS}

Laser direct write surface texturing can provide a quick and easy way to modify metal surfaces and improve the growth and adhesion of cells. Large scale groove features including depth and width can be controlled and predicted with simple equations. Groove width scales with laser pulse energy and is mostly independent of other parameters, therefore, groove width can be established by modifying laser pulse energy. The depth of a groove can be independently manipulated within bounds by modifying translation distance between pulses. If deeper grooves are needed multiple passes can be used to incrementally increase groove depth. Secondary groove features also have a profound effect on the proliferation of cells on the surface. These features are further manipulated using lower energy finishing passes with only a minor impact upon overall groove shape. Modifying the translation distance between secondary passes can change the spacing between secondary ridges perpendicular to the main groove, while modifying pulse energy of the final pass can change the abundance and shape of submicroscale splatter. Higher energy passes create more abundant pyres, bubbles, and features parallel to the groove. Machining final passes in liquid environments enhances the ability to modify surfaces using final passes. In addition to surface texturing, laser processing can cause changes to the material stoichiometry. Preferential evaporation of aluminum from molten regions after ablation leads to depletion in the base of the grooves, but enhancement near the tops of the ridges. However, these effects can be ameliorated by machining under liquids.

The culmination of these studies, designing and implementing multiple grooves with the same basic parameters and varied secondary parameters demonstrates that this method is valid and can be used to generate carefully engineered grooves. Final cell studies show that the type of secondary groove parameters formed using this method do have a significant effect on cell growth. With this large set of tools, direct write laser texturing holds significant potential for surface optimization in order to improve osseoeintegration for structural implants.

\section{ACKNOWLEDGMENTS}


The authors acknowledge financial support for this project from the NSF through the US-Africa international materials institute at Princeton University.

\section{REFERENCES}

1. Meyer U, Buchter A, Wiesmann HP, Joos U, Jones DB: European Cells and Materials 2005. 9: p. 39-49.

2. Cochran DL, Buser D, ten Bruggenkate CM, Weingart D, Taylor Tm, Bernard J-P, Peters F, Simpson JP: Clinical Oral Implant Research, 2002. 13: p. 144.

3. Roccuzzo M, Bunino M, Priglio F, Bianchi S: Clinical Oral Implants Research, 2001. 12: p. 572.

4. E. T. den Braber, J. E. de Ruijter L. A. Ginsel A. F. von Recum J. A. Jansen: Journal of Biomedical Materials Research, 1998. 40(2): p. 291-300.

5. MD Ward, DA Hammer: Biophysics Journal, 1993. 64: p. 936-959.

6. N. Wang, J.P. Butler, and D.E. Ingber: Science, 1993. 260: p. 1124-1127.

7. Hohn HP, Steih U, Denker HW: In Vitro Cell Developmental Biology, 1995. 31A: p. 37-44.

8. den Braber, E. T., J. E. de Ruijter, H. T. J. Smits, L. A. Ginsel, A. F. von Recum, and J. A. Jansen: Biomaterials, 1996. 17(11): p. 1093-1099.

9. A. Khakbaznejad, B. Chehroudi D. M. Brunette: Journal of Biomedical Materials Research Part A, 2004. 70A(2): p. 206-218.

10. W. O. Soboyejo, B. Nemetski S. Allameh N. Marcantonio C. Mercer J. Ricci: Journal of Biomedical Materials Research, 2002. 62(1): p. 56-72.

11. Britland, Stephen, Peter Clark, Patricia Connolly, and Geoffrey Moores: Experimental Cell Research, 1992. 198(1): p. 124-129.

12. X.F. Walboomers, H. J. E. Croes L. A. Ginsel J. A. Jansen: Journal of Biomedical Materials Research, 1999. 47(2): p. 204-212.

13. Dalby, Matthew John, Mathis O. Riehle, Duncan S. Sutherland, Hossein Agheli, and Adam S. G. Curtis: Biomaterials, 2004. 25(23): p. 5415-5422.

14. Pedraza AJ, Fowlkes JD, Lowndes DH: Applied Physics Letters, 1999. 74: p. 2322-2324.

15. Her, T. H., R. J. Finlay, C. Wu, and E. Mazur: Applied Physics A: Materials Science \& Processing, 2000. 70(4): p. 383-385.

16. Long-Hao Li, Kong Young-Min, Kim Hae-Won, Kim Young-Woon, Kim Hyoun-Ee, Heo Seong-Joo, Koak Jai-Young: Biomaterials, 2004. 25: p. 2867-2875.

17. Sauli Kujala, Ryhanen J, Danilov A, Tuukkanen J: Biomaterials, 2003. 24: p. 4691-4697.

18. Bellow CG, Heersche JNM, Aubin JE: Calcification Tissue Int., 1999. 61(1): p. 59-65. 